\title{
UN INTENTO DE MODELIZAR EL COMPORTAMIENTO ELECTORAL. EL CASO DE LA COMUNIDAD VALENCIANA ENTRE 1983 Y 1987
}

\author{
Juan Miguel Albertos Puebla \\ Departamento de Geografía. Facultad de Geografía e Historia Universidad de Valencia
}

\section{RESUMEN}

El presente artículo explora la posibilidad de modelizar el comportamiento electoral, tomando como caso de estudio la evolución de los municipios de más de 2.000 habitantes en la Comunidad Valenciana entre 1983 y 1987. En concreto, se confirma la posibilidad de aplicar modelos de difusión de innovaciones, al comprobarse la existencia de niveles de saturación en el voto a las distintas opciones políticas, o la transmisión de los nuevos comportamientos electorales a través del sistema de ciudades en un proceso de filtraje hacia abajo.

\begin{abstract}
This work explores our possibilities to model the electoral behaviour, using as a case-study the evolution of municipalities bigger than 2.000 inhabitants in the Comunidad Valenciana between 1983 and 1987. Specifically, we confirm that we are found saturation levels in the vote to a political option, and the transmission of new electoral behaviours within the city-system, across a filtering-down process.
\end{abstract}

\section{Introducción}

Tratamos con el presente artículo de aportar algunas ideas en torno a las posibilidades de modelización que, si optamos por trabajar sobre una dimensión espacial, ofrece el comportamiento electoral. Pensamos que una aproximación de este tipo nos puede ayudar a comprender las pautas que sigue el comportamiento del voto a los distintos partidos bajo condiciones de estabilidad del mapa político a medio plazo, así como a conocer mejor la forma en que tienen lugar los procesos de cambio gradual de los resultados electorales. Debe quedar claro que la perspectiva de este trabajo no es el análisis sociológico de las causas del comportamiento electoral, ni de su sentido, ni de su alteración. Lo que pretendemos es dejar de manifiesto la existencia en abstracto de una serie de regularidades en el 


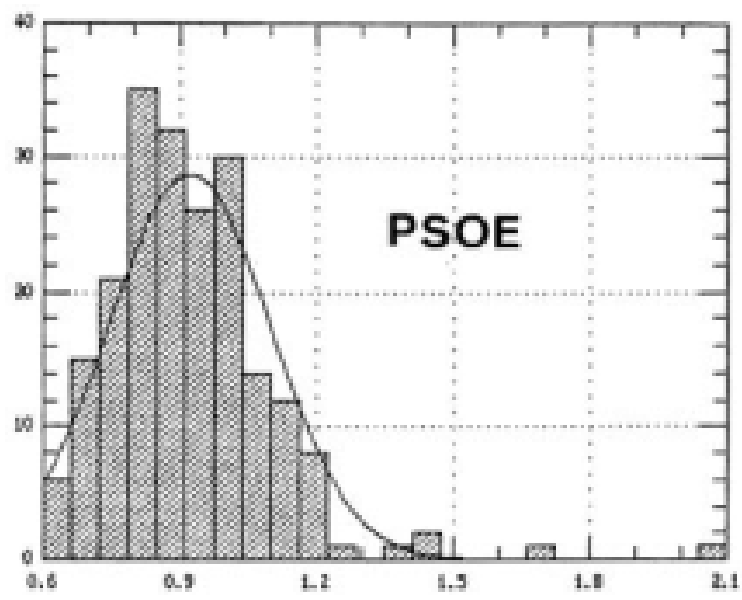

\section{Cociente entre votos en 1987 y votos en 1983}

GRÁFICO 1. Distribución de frecuencias de los cocientes votos en 1987/votos en 1983, para el PSOE y el CDS, y ajuste a la distribución normal. 206 municipios de más de 2000 habitantes.

comportamiento electoral que tienen que ver con la estructura de asentamientos de la población, así como con los canales de difusión de información.

Creemos que la relativa lejanía en el tiempo de los procesos electorales elegidos para este estudio, -entre 1983 y 1987-, nos permite abordar el tema con un distanciamiento mayor, del que sería posible si aludiéramos a consultas más cercanas a nosotros. Además, se trata de una época ideal para nuestro análisis en tanto que está caracterizada por una cierta estabilidad de los comportamientos, al tiempo que es posible apreciar, sin embargo, algunos cambios incipientes en curso, como son la pérdida de votos, ligera pero constante, de las opciones mayoritarias -PSOE y AP-, y el consiguiente incremento de los partidos con menor representación -UV, CDS, y IU-.

\section{Metodología y resultados}

Hemos tomado como caso de estudio la Comunidad Valenciana, trabajando sobre los resultados a escala municipal de las elecciones autonómicas de 1983, generales de $1986 \mathrm{y}$, de nuevo, autonómicas de 1987. Para llevar a cabo nuestro análisis se ha construido una base de datos en hoja LOTUS 123, con los resultados municipales de las tres consultas 


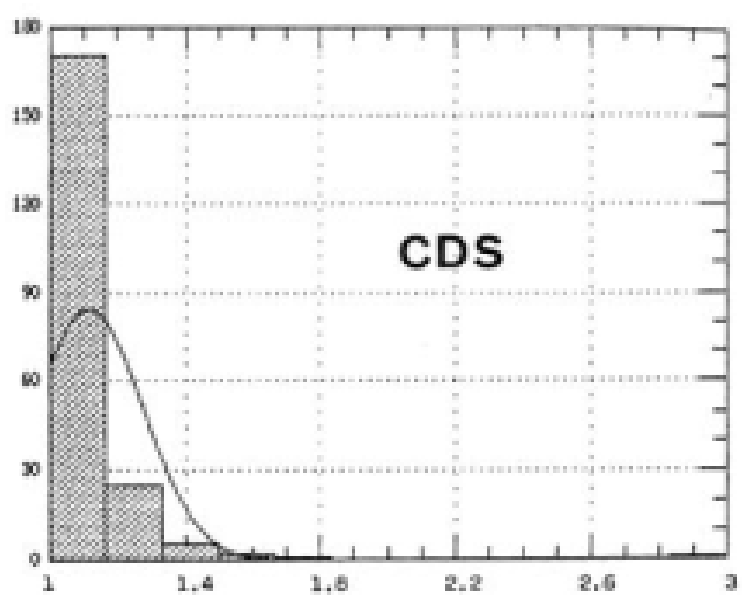

Cociente entre votos en 1987 v votos en 1983

GRÁFICO 1 (continuación). Distribución de frecuencias de los cocientes votos en 1987 / votos en 1983, para el PSOE y el CDS, y ajuste a la distribución normal. 206 municipios de más de 2000 habitantes.

electorales, así como el nombre y tamaño del municipio. El tratamiento estadístico se ha llevado a cabo con el programa STATGRAPHICS de STSC.

Como primer paso, se ha realizado un análisis de las variables con las que trabajamos, lo que nos permitirá el uso de instrumentos estadísticos adaptados a sus características. La variación del apoyo que recibe un determinado partido entre una y otra elección podemos medirla tanto por la diferencia absoluta de votos entre uno y otro momento, como por el cociente entre ambas cifras. Las diferencias de votos están fuertemente correlacionadas con el tamaño del municipio, y presentan una distribución asimétrica de sesgo negativo, que en modo alguno puede asimilarse a una distribución normal. Por su parte, como puede apreciarse en el GRÁFICO 1, los cocientes presentan también una distribución asimétrica, negativa en este caso, lejos también de la distribución normal. Por todo ello, nos vemos obligados, en cualquier caso, al uso de métodos estadísticos no paramétricos. Además, cuando queramos emplear los cocientes habremos de limitar el trabajo a los 206 municipios de más de 2000 habitantes (de un total de 533), que constituyen la inmensa mayoría del censo; el comportamiento profundamente errático en este caso de los municipios menores de 2000 habitantes es lo que ha motivado su exclusión del estudio. 
Hemos empleado la información recopilada en dos tipos de análisis:

1) por un lado, hemos relacionado las variaciones de voto de cada partido, y, en particular del PSOE, con las variaciones de voto de los restantes partidos, y la evolución de la abstención. Ello nos ha de permitir hacemos una idea en torno a los flujos de votos que se producen entre las distintas opciones políticas.

2) por otro lado, hemos asimilado la adopción de un determinado comportamiento electoral a un modelo general de difusión de innovaciones. Según esto, el nivel de adopción de un determinado comportamiento electoral, así como su ritmo de crecimiento, estaría en función de que se hubiese o no alcanzado el que podemos denominar su nivel de saturación. Desde otro punto de vista, la adopción de las innovaciones de comportamiento deberían poder ponerse en relación con las estructuras que condicionan la difusión de la información, esto es, las relaciones de jerarquía entre los distintos tamaños de núcleo, y las relaciones de vecindad entre núcleos cercanos ${ }^{1}$.

\section{III.1. Los flujos de votos entre partidos}

De los resultados electorales es imposible obtener una matriz completa de pérdidas y ganancias de votos de cada partido en relación con el resto. Sin embargo, podemos aproximarnos bastante a lo que está sucediendo en este campo a través de métodos indirectos. Para ello hemos hallado los coeficientes de correlación de rangos de Spearman de las diferencias de votos entre 1983 y 1987 para los 533 municipios de la Comunidad tomando parejas de partidos. En la TABLA 1 presentamos las correlaciones entre los partidos que compiten por el espacio electoral que detenta el PSOE.

Tabla 1

COEFICIENTES DE CORRELACIÓN DE RANGOS DE SPEARMAN DIFERENCIAS DE VOTOS ENTRE 1983 Y 1987

\begin{tabular}{lcccc}
\hline & PSOE & IU & UV & CDS \\
\hline IU & -0.302 & & & \\
UV & -0.378 & 0.336 & & \\
CDS & -0.441 & 0.268 & 0.547 & \\
ABSTENCIÓN & $-0.025\left(^{*}\right)$ & -0.118 & -0.113 & -0.092 \\
\hline
\end{tabular}

(*) Coeficiente no significativo al $5 \%$

Se demuestra que la caída de los votos del PSOE entre 1983 y 1987 está bien relacionada con el incremento del voto del resto de los partidos, y, especialmente, del incremento del voto al CDS, mientras que no aparece apenas relacionada con la evolución de la abstención. Podemos suponer que, en líneas generales, los votos que pierde el PSOE no van a engrosar la abstención, sino que se dirigen hacia otras opciones políticas.

\footnotetext{
${ }^{1}$ Torsten Hägerstrand, 1966, «Aspects of the Spatial Structure of Social Communications and the Diffusion of Information», Papers of the Regional Association, 16, pp. 27-42.
} 
IU, UV, y CDS muestran correlaciones positivas entre sí, lo que indicaría que, al menos por el momento, no existía competencia entre ellos por el espacio electoral, aunque todos la mantenían con el PSOE. Sin embargo, al final del período, entre 1986 y 1987 sí que comienza a aparecer un coeficiente de correlación negativo entre las variaciones del voto de UV y del CDS, lo que estaría indicando que ya empezaba a producirse cierta competencia entre ambas fuerzas por un mismo espacio electoral.

Si el análisis del comportamiento de las variaciones del voto dirigido al PSOE lo realizamos para el período que va de 1986 a 1987, los resultados obtenidos son algo diferentes. De nuevo, la variación del voto del PSOE se relaciona con signo negativo con la del resto de partidos antes estudiados, pero, ahora, también lo está con la evolución seguida por la abstención. La explicación de este fenómeno quizás esté en el distinto carácter de las consultas electorales de uno y otro año. Las de 1986 fueron elecciones generales, con un nivel de participación claramente mayor que las de 1987, de carácter autonómico. El incremento de la abstención entre ambas fechas parece que se podría explicar, al menos parcialmente, por la desmovilización de parte del espacio electoral del PSOE que, ante una consulta considerada de menor rango decisorio, opta por no votar.

Por su parte las variaciones de voto de AP, que también desciende en número de votos entre 1983 y 1987, se correlacionan sobre todo, y como era de esperar, con la evolución seguida por $\mathrm{UV},(\mathrm{r}=-0.651)$, pero también, aunque en menor medida, con la del CDS ( $\mathrm{r}=-0.241)$. Por tanto, ha sido UV la mayor beneficiaria del descenso electoral de AP, aunque también el CDS ha aumentado a su costa, en cierta medida, su número de votos.

\section{III.2. La variación del voto como un proceso de difusión de innovaciones}

Es posible asimilar el proceso de adopción de un determinado comportamiento electoral a un modelo de difusión de innovaciones. Las variables que intervienen en un proceso de difusión de innovaciones ${ }^{2}$-acceso a información y grado de resistencia a la adopción-, son también aplicables a las variaciones en el voto.

En esta línea, si suponemos que cada partido, dentro de una situación de estabilidad a medio plazo del mapa político, tiene un espacio electoral máximo que puede cubrir, es de esperar que, de forma paulatina, sus resultados en las distintas localidades irán ajustándose a este nivel de tendencia, descendiendo en aquellas en las que se encontrara por encima, y subiendo donde se hallara por debajo. Este concepto, que denominaremos nivel de saturación, encuentra su paralelo en la teoría de difusión de innovaciones en el nivel máximo de adopción, punto hacia el que la curva de adopción tiende de forma asintótica una vez que el incremento de nuevos adoptadores se hace de forma extremadamente lenta, cuando el proceso de difusión ha entrado en su última fase ${ }^{3}$.

La determinación de este nivel de saturación, a partir del cual es difícil obtener nuevos incrementos en los resultados de un partido sin que tengan lugar profundos cambios en la situación política general, puede tener un gran interés, en tanto que muestra los lugares en los que es posible todavía esperar cierto crecimiento. Hemos intentado hallar cuáles son estos niveles de saturación para los distintos partidos. Para ello se ha calculado la correlación existente entre los votos obtenidos en 1983, y el cociente resultante de comparar los votos obtenidos en 1983 y 1987, que nos indica la tasa de crecimiento o decrecimiento de

\footnotetext{
${ }^{2}$ Herbert G. Kariel, y Patricia E. Kariel, 1972, Explorations in Social Geography, Reading (Mass.), AddisonWesley Publishing Company.

${ }^{3}$ Lawrence A. Brown, 1981, Innovation Diffussion: A New Perspective, Nueva York, Methuen.
} 
los votos a cada partido. En este caso hemos aplicado el procedimiento a los 206 municipios de más de 2000 habitantes, evitando así la distorsión que proviene del comportamiento errático de los núcleos de población más pequeños, especialmente acusado al usar el método de cocientes. En general, la mejor línea de ajuste se obtiene con ecuaciones del tipo

$$
Y=A * X^{B}
$$

donde $Y$ es el incremento de votos del partido como cociente entre los resultados de 1987 y 1983 , y $X$ es el porcentaje de votos de 1983 sobre el total de votos emitidos. El GRÁFICO 2 muestra de forma detallada el ajuste realizado para el caso del PSOE, mientras que la TABLA 2 presenta los resultados generales para el conjunto de los partidos.

Tabla 2

\begin{tabular}{|c|c|c|c|c|}
\hline Partido & $\begin{array}{l}\text { Coeficiente } \\
\text { Correlación }\end{array}$ & $\begin{array}{r}\text { Varianza } \\
\text { Explicada } \\
\end{array}$ & Pendiente & $\begin{array}{c}\text { Probab. } \\
\text { Hipot. Nula }\end{array}$ \\
\hline PSOE & -0.606 & $36.7 \%$ & -0.46 & 0.00 \\
\hline IU & -0.761 & $57.9 \%$ & -0.59 & 0.00 \\
\hline AP & -0.026 & $0.1 \%$ & -0.05 & 0.70 \\
\hline CDS & -0.661 & $43.6 \%$ & -0.64 & 0.00 \\
\hline
\end{tabular}

El ajuste no pudo aplicarse a UV por no tener votos por separado en 1983 -se presentaba en coalición con AP-, lo que origina que sus tasas de crecimiento tomen un valor infinito. Por lo demás, puede decirse que el ajuste obtenido es muy bueno para todos los partidos, que presentan una proporción de varianza explicada realmente alto, con la excepción de AP. En este caso, la salida de UV de la Coalición Popular ha debido introducir un elemento de profunda distorsión en la variación del voto experimentada por esta opción política, lo que nos impide asimilar su comportamiento al modelo.

El incremento de votos de cada partido entre 1983 y 1987 aparece estrecha e inversamente relacionado con el porcentaje obtenido al principio del período. El signo negativo del coeficiente nos está indicando que los incrementos de voto son mayores allí donde el porcentaje de votos inicial era más pequeño, y que son menores, o que incluso se producen descensos, allí donde se partía de una situación de apoyo más amplio. Podemos establecer en el valor de $Y=1$ el punto que indica un mantenimiento del apoyo electoral en el volumen que alcanzó a principios del período. A este valor de $Y$, le corresponderá un valor de $X$, que llamaremos $X_{\mathrm{s}}$, que podemos considerar como el nivel de saturación de ese partido, o espacio electoral máximo que puede llegar a alcanzar sin cambios profundos en el mapa político general, y que equivale a

$$
\mathrm{X}_{\mathrm{s}}=(1 / \mathrm{A})^{(1 / \mathrm{B})}
$$

En la TABLA 3 se ofrecen los valores que adopta $X_{s}$ para los diferentes partidos 


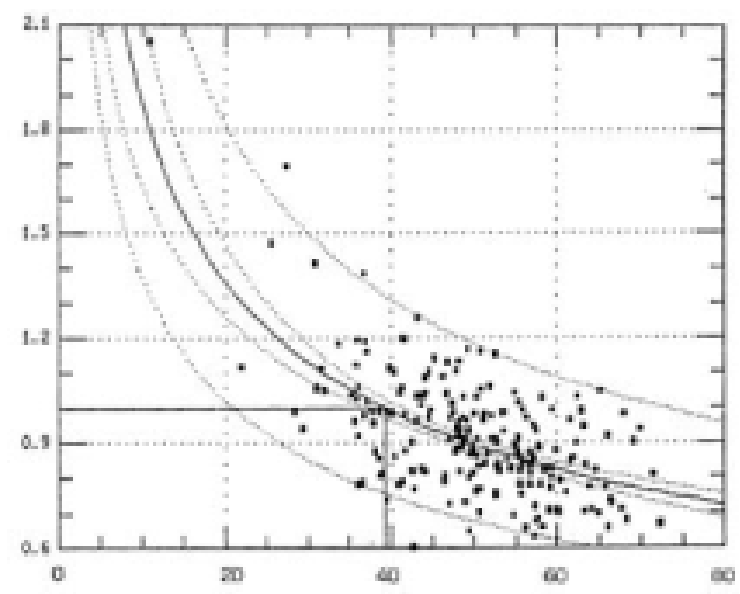

Porcentaje de votos en 1983

GRÁFICO 2. Diagrama de puntos representando la evolución del voto al PSOE entre 1987 y 1983, en función del voto obtenido en 1983, y ajuste de curva exponencial. 206 municipios de más de 2000 habitantes.

Tabla 3

NIVELES DE SATURACIÓN EN EL VOTO

\begin{tabular}{lc}
\hline Partidos & Valor $\mathbf{X}_{\mathbf{s}}(\boldsymbol{\%})$ \\
\hline PSOE & 39.2 \\
IU & 8.8 \\
CDS & $12.9(*)$ \\
AP & - \\
UV & - \\
*) Calculado con una ecuación del tipo $Y=A X+B$
\end{tabular}

Según nuestro análisis los resultados de cada partido en las diferentes localidades tenderá a acercarse tras sucesivas consultas electorales a este nivel de saturación. Hay que entender, no obstante, que el modelo sólo explica una parte de la varianza, aunque ésta sea 
muy grande. Hay otros factores que juegan y que deben tenerse en cuenta a la hora de explicar el comportamiento de cada municipio en concreto: tradiciones locales, gestión local de los diferentes partidos, etc. Sin embargo, este proceso de asimilación progresiva de los comportamientos locales a una norma general, puede ponerse en relación con un proceso de difusión de ideas, en el que existen importantes diferencias en el comportamiento de los distintos municipios, según respondan de forma más temprana o tardía a los cambios de comportamiento electoral. Los núcleos más alejados de los principales canales de información tardarían más en adecuar sus actitudes a la norma general, y de ahí, que se produzcan esos movimientos compensatorios, por encima y por debajo del nivel de saturación de cada partido en las sucesivas consultas electorales.

Resulta, por tanto, de gran interés comprender cómo se produce el proceso de difusión de ideas entre los diferentes núcleos de población. Si admitimos la existencia de un paralelismo entre éste y los procesos de difusión de innovaciones, dos son los procesos implicados, que suelen actuar de forma complementaria ${ }^{4}$ :

A) las relaciones de carácter jerárquico, según las cuales las ciudades más grandes, que tienen un mayor acceso a información, adoptarían antes los cambios en el comportamiento electoral, de forma que, a partir de ellas, éstos desciendan por toda la escala urbana, en un proceso de filtrado hacia abajo, que afecta tanto a la información como a la propia adopción del nuevo comportamiento.

B) las relaciones de vecindad, que propician la adopción del nuevo comportamiento por cercanía física con un adoptante previo, circunstancia que intensifica las relaciones y, con ellas, el intercambio de información.

En este trabajo nos centraremos en la comprobación de la tesis que hace referencia a la existencia de canales de difusión de estructura jerárquica, en relación con el tamaño de municipio, que afectan a la adopción más o menos temprana de los nuevos comportamientos electorales. Para ello, tomaremos como ejemplo de estudio la evolución de porcentaje de votos que recibe el PSOE. El descenso en el apoyo que recibe el PSOE es la característica más notable del período estudiado, que se extiende, además, por todo el territorio, variando sólo de unas áreas a otras el destino de los votos que pierde el PSOE (otros partidos, o la abstención).

La TABLA 4 y el GRÁFICO 3 dejan bien clara cual ha sido la evolución seguida:

Tabla 4

PORCENTAJE DE VOTOS DEL PSOE SEGÚN TAMAÑOS DE MUNICIPIO

\begin{tabular}{lccc}
\hline Tamaño & $\mathbf{1 9 8 3}$ & $\mathbf{1 9 8 6}$ & $\mathbf{1 9 8 7}$ \\
\hline $2000-2999$ & $48.2 \%$ & $45.9 \%$ & $43.8 \%$ \\
$3000-4999$ & $47.3 \%$ & $46.1 \%$ & $41.4 \%$ \\
$5000-9999$ & $48.7 \%$ & $46.6 \%$ & $41.4 \%$ \\
$10000-24999$ & $52.3 \%$ & $49.8 \%$ & $43.6 \%$ \\
$25000-49999$ & $54.0 \%$ & $51.0 \%$ & $43.2 \%$ \\
$50000-399999$ & $56.0 \%$ & $47.6 \%$ & $40.7 \%$ \\
más de 400000 & $46.6 \%$ & $39.7 \%$ & $34.8 \%$ \\
\hline
\end{tabular}

\footnotetext{
${ }^{4}$ Poul Ove Pedersen, 1970, «Innovation Diffusion within and between National Urban Systems», Geographical Analysis, vol. 2, pp. 203-254.
} 
Puede observarse como en el período estudiado se produce un descenso generalizado del voto al PSOE en todos los tamaños de municipio. Sin embargo, la caída no tiene lugar con la misma intensidad en todos los lugares, sino de una forma muy sesgada según el tamaño del municipio. En 1983 el máximo de apoyo se obtiene en los municipios mayores de 50000 habitantes, con la relevante excepción de la ciudad de Valencia, y, a partir de ahí, éste desciende con el tamaño del municipio. En 1986 el máximo apoyo se obtiene en el estrato entre 25000 y 50000 habitantes, y en 1984 éste pasa a conseguirse en los núcleos entre 10000 y 25000. Paralelamente, la pérdida de votos, que en las ciudades de más de 50000 habitantes oscila entre 12 y 16 puntos, es sólo de 4 puntos en los municipios entre 2000 y 3000 habitantes.

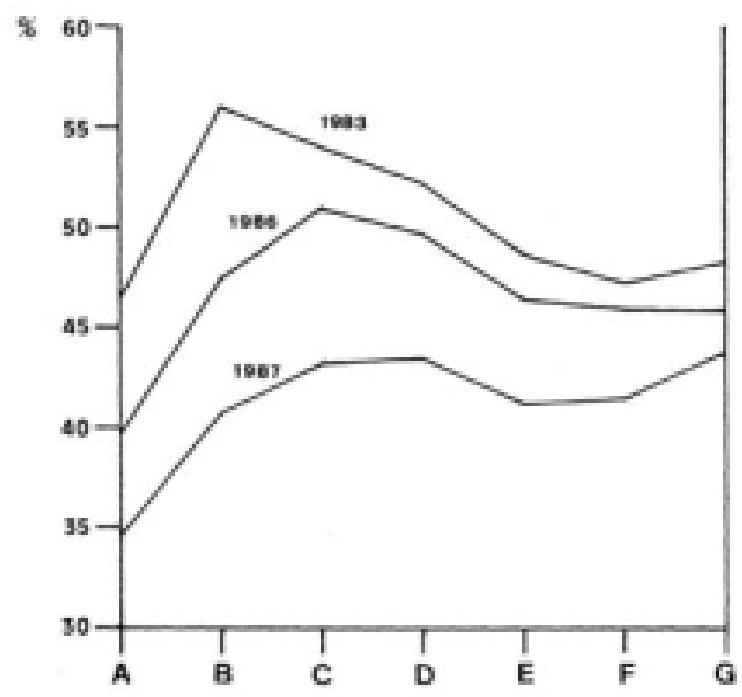

GRÁFICO 3. Porcentaje de votos al PSOE respecto al total de votos emitidos, en función del tamaño de municipio, en 1983, 1986, y 1987. 206 municipios de más de 2000 habitantes.

A) más de 400000; B) 50000-399999; C) 25000-49999;

D) 10000-24999; E) 5000-9999; F) 3000-4999; G) 2000-2999 
Todo parece indicar que se está produciendo una especie de movimiento ondulatorio, en función del cual el nuevo comportamiento electoral, -en este caso el descenso de votos del PSOE-, que se inicia en las grandes ciudades, se va difundiendo por todo el territorio a través de la jerarquía urbana, afectando a cada municipio de una forma más temprana y con una mayor intensidad, cuanto mayor sea su tamaño. Hasta tal punto esto es así, que el nivel de apoyo que recibe el PSOE, en 1983 básicamente descendente con el tamaño de núcleo, en 1987 empieza ya a presentar síntomas del comportamiento inverso, esto es, mostrando un apoyo vagamente ascendente a medida que disminuye el tamaño de núcleo. La tesis que mantiene la existencia de una componente jerárquica en los procesos de difusión de los cambios en los comportamientos electorales parece, pues, corroborada por los hechos.

\section{Conclusiones}

Pensamos que, con todo lo expuesto más arriba, queda claramente patente que existe la posibilidad de modelizar determinados aspectos del comportamiento electoral en su dimensión espacial. En este sentido, los modelos de difusión de innovaciones, que basan su funcionamiento en el acceso de los potenciales adoptadores a información sobre la innovación, pueden ser utilizados con cierto éxito para este fin.

En concreto, creemos que es posible, en condiciones de estabilidad del comportamiento político, utilizar el concepto de nivel de saturación, para definir el tope máximo probable que puede alcanzar el voto a un determinado partido, esto es, la adopción máxima de ese comportamiento. Sobre esta base puedan establecerse hipótesis en la línea de intentar predecir, en los casos de las localidades que se encuentren por debajo, o por encima, de ese nivel, la evolución futura más probable, tanto en relación al sentido de las posibles alteraciones del voto, como a la velocidad del proceso.

En esta línea, tiene también un gran interés nuestra constatación de la validez de los esquemas de transmisión de la información y de las ideas a través de la jerarquía urbana, en su aplicación a la difusión de los comportamientos electorales. Las innovaciones en las actitudes frente al voto parecen seguir el mismo camino, en un proceso de filtrado hacia abajo a través del sistema de ciudades, que el resto de las innovaciones sociales o económicas.

Debe quedar claro, por último, que existen otras muchas variables que condicionan tanto el nivel de voto de cada partido, como su probable evolución, en relación sobre todo con la estructura social y económica de cada área. Deliberadamente, hemos hecho abstracción de ellas, puesto que nuestro propósito era dejar patente la existencia de comportamientos que atienden, en buena medida, a la disposición y la estructura de los canales de transmisión de información, que tienen que ver, en última instancia, con la organización misma del sistema urbano. 